\title{
Do general relativistic effects limit experiments to test the universality of free fall and the weak equivalence principle?
}

\author{
Anna M. Nobili \\ Department of Physics “E. Fermi,” University of Pisa, Largo B. Pontecorvo 3, 56127 Pisa, Italy \\ and INFN-Istituto Nazionale di Fisica Nucleare, Sezione di Pisa, Largo B. Pontecorvo 3, 56127 Pisa, Italy
}

(Received 11 August 2016; published 28 December 2016)

\begin{abstract}
The universality of free fall and the weak equivalence principle, which are at the basis of general relativity, have been confirmed to 1 part in $10^{13}$. Space experiments with macroscopic test masses of different composition orbiting Earth inside a low altitude satellite aim to improve this precision by 2 orders of magnitude (with the Microscope satellite launched on April 25, 2016) and up to 4 orders of magnitude (with the Galileo Galilei satellite). At such a high precision, many tiny effects must be taken into account in order to be ruled out as the source of a spurious violation signal. In this work, we investigate the general relativistic effects, including those which involve the rotation of both Earth and the test masses, and show that they are by far too small to be considered even in the most challenging experiment.
\end{abstract}

DOI: $10.1103 /$ PhysRevD.94.124047

\section{INTRODUCTION}

The universality of free fall (UFF), also known as the weak equivalence principle (WEP), refers to the fact that in a gravitational field, all bodies fall with the same acceleration regardless of their mass and composition (see, e.g., [1]). As stated by Einstein [2] in 1916, the general theory of relativity (GR) requires this fact to hold.

The best experimental tests so far involve artificial proof masses suspended on rotating torsion balances [3-5] or celestial bodies (Earth and the Moon through the Lunar Laser Ranging experiment [6-8]). They show no anomalous differential acceleration between the falling bodiesin the fields of Earth and the Sun - to about $10^{-13}$.

Considerable improvements are expected in the field of Earth by flying macroscopic proof masses inside a low altitude spacecraft. The Microscope satellite [9] launched in April 2016 aims at $10^{-15}$ and Galileo Galilei (GG) [10] aims at $10^{-17}$. As for cold atom tests, at present they have reached a few $10^{-8}[11]$ and are not expected to compete with high-precision space tests based on macroscopic bodies [12].

Despite their high-precision goals and, consequently, the very small effects to be measured, the GG and Microscope experiments are designed and investigated within Newtonian mechanics on the assumption that general relativistic effects are too small to compete with the sought for violation signal.

During a recent competitive selection of proposals short listed for the medium size mission M4 of the European Space Agency (ESA), this assumption was questioned by the Science Assessment Review Panel (SARP) appointed by ESA to evaluate the GG proposal. From their report on GG [13], we read

"The breakdown of the WEP is sought in the frame work of the response of test matter to terrestrial Newtonian gravitation. The source of terrestrial Newtonian gravitation is independent of the Earth's (non uniform) rotation. Furthermore, the test cylinders in the proposed experiment are spinning. In General Relativity the gravitational field of a spinning source depends on its spin. Also the mass centroid motion of extended spinning test matter in an external gravitational field may depend on its spin and still be geodesic (independent of inertial mass) when its spin is zero. The estimates, based on General Relativity, of the effect of the Earth's rotation on the motion of each spinning cylinder or the laser interferometer and their relevance to the interpretation of any non null signal at the expected level of accuracy have not been sufficiently explained to the satisfaction of the SARP".

Experimental evidence of a violation of UFF/WEP would require either a modification/augmentation of GR or the existence of a new composition-dependent force of nature. Either way, it would make a revolution in physics. On the other hand, a null result to a very high precision will be a landmark for any attempt at overcoming the current physics impasse. The situation is reminiscent of that at the end of the 19th century, when Michelson and Morley [14] tested by very precise light interferometry the propagation of the newly discovered electromagnetic waves through the ether and proved that the ether does not exist; a very precise null result, which in 1905 led to the theory later named special relativity (Michelson was awarded the Nobel Prize in 1907).

By reaching its goal, Microscope will improve the current best tests of UFF/WEP by 2 orders of magnitude, to $10^{-15}$. Should the possibility of a nonzero violation signal emerge from Microscope data, it will call for urgent checking, and more precise space experiments such as GG (which aims at $10^{-17}$ ) might become of interest to space agencies. It is, therefore, important and timely to firmly 
establish the role of general relativistic effects in highprecision satellite tests of UFF/WEP.

Deviations from Newtonian predictions in the motion of orbiting bodies have been investigated since the birth of general relativity in the hope to provide observational evidence for the new theory. In November 1915, Einstein presented to the Prussian Academy of Sciences in Berlin his results on the "Explanation of the Perihelion Motion of Mercury from the General Theory of Relativity" [15], and one week later, "The Field Equations of Gravitation" [16]. A year later De Sitter presented to the Royal Netherlands Academy of Arts and Sciences the work: "Planetary motion and the motion of the moon according to Einstein's theory", published in 1917 [17].

Einstein's Mercury paper and de Sitter's work mentioned above deal with nonrotating masses. However, all celestial bodies rotate. According to Newton, the gravitational field of a celestial body does not depend on whether the body rotates or not. Not so in general relativity. In 1918, Thirring and Lense [18,19] calculated the secular effects of the rotation of the central body on the orbits of planets and moons (see the English translation and comments on the Thirring and Lense papers by [20]).

Since then, considerable theoretical work has been carried out to include also the rotation of the secondary body, leading to the so-called Mathisson-Papapetrou-Dixon equations [21-23]. The whole subject was revisited in the early 1960s [24-26] with the proposal to fly a highprecision gyroscope in low Earth orbit in order to measure general relativistic effects (the GP-B mission launched in 2004 [27]) and in the 1970s became of primary importance for understanding binary systems made by very compact rapidly rotating stars $[28,29]$.

Similar to the modern torsion balances used for testing UFF/WEP, the Microscope and GG satellites are designed to rotate in order to up-convert the target signal to higher frequency where important noise sources are known to be smaller than they are at lower frequencies [30,31].

We compute the general relativistic effects in these experiments by referring to the literature available in which the spin angular momentum of both the primary and secondary body are taken into account. We refer in particular to the work of Barker and O'Connel $[28,29]$ (in checking the equation numbers quoted, please note that they refer to the primary and secondary body with the numbers 2 and 1, respectively, while in this work we do the opposite).

This paper is organized as follows. In Sec. II we estimate the differential accelerations between the test masses as predicted by GR, in the absence of spin as well as in the presence of a spinning Earth and of spinning test cylinders. We also recall the Newtonian differential effects due to the quadrupole mass moments of the interacting bodies. Section III deals with differential precession of the orbits due to general relativity (and Newtonian dynamics) and relates them to the differential accelerations which give rise to them and might compete with the violation signal. In Sec. IV the general relativistic and Newtonian effects on the spin axes of the test cylinders are estimated. In Sec. V we discuss the effects of rotation on the laser interferometry readout of GG. The conclusion that, in all cases, general relativistic effects are negligible by and large, is drawn in Sec. VI.

\section{GENERAL RELATIVISTIC ACCELERATIONS COMPETING WITH A VIOLATION SIGNAL}

Tests of the universality of free fall are quantified by the fractional differential acceleration

$$
\eta=\frac{\Delta a}{a}
$$

between two test masses of different composition as they fall in the gravitational field of a source body with the average acceleration $a$ ("driving signal"). The physical observable is the differential acceleration $\Delta a$ of the falling masses relative to each other, pointing to the center of mass of the source body.

For test masses orbiting Earth inside a low altitude, low eccentricity, sun-synchronous satellite such as Microscope or GG, a violation signal $(\Delta a \neq 0)$ driven by Earth would have the orbital frequency, while the driving signal $a$ at the denominator is the gravitational acceleration $g(h)$ caused by Earth at the satellite altitude $h ; h \simeq 630 \mathrm{~km}$ for GG (with $g(h) \simeq 8.1 \mathrm{~ms}^{-2}$ ) and slightly higher for Microscope.

Up-conversion of the signal to higher frequency (the higher, the better) is regarded by all experimentalists as a crucial asset because thermal and electronic noise are lower at higher frequency. This fact has been demonstrated by the rotating torsion balances, which have been able to reach the thermal noise limit expected at their rotation rate (see [30], Fig. 20).

For this reason, both Microscope and GG are designed to rotate, though the way they accomplish it is different due to the different experiment design. The test masses are (nominally) concentric hollow cylinders in both cases.

A very good coincidence of the centers of mass is crucial because of a major Newtonian effect caused by offcentering. Because of the nonuniformity of the gravitational force, there is a tidal differential acceleration from Earth (nonzero gravity gradient); it acts at twice the orbital frequency, but there is also a smaller tidal effect proportional to the eccentricity of the orbit, which acts at the orbital frequency and, therefore, competes directly with the sought for violation signal. This is a major limiting factor to space tests of UFF, which cannot be totally eliminated because it is impossible to inject the satellite in an exactly circular orbit. Indeed, for high-precision tests, the centers of mass of the test cylinders must be centered on one another far better than it is typically achieved at the time of launch by construction and mounting. 
In the Microscope satellite, the test cylinders are required to be concentric within $20 \mu \mathrm{m}$ at launch, and no further adjustment is performed in space. The Microscope scientists plan to use the tidal effect at twice the orbit frequency within a posteriori data analysis in order to reduce-from the measurement of this effect- the unknown level of offcentering between the test cylinders to within $0.1 \mu \mathrm{m}$, i.e., a factor 200 better than achieved via hardware at launch (see [9], p. 4). A recent press release by the French space agency reports measured offsets of 25 and $33 \mu \mathrm{m}$ [32] (the two values are likely to refer to the two accelerometers carried by the Microscope satellite, each one with two test cylinders).

In the Microscope satellite, each cylinder is constrained to move along its symmetry axis (sensitive axis): weak electrostatic coupling along the axis, 1 degree of freedom, and the effect of the violation signal maximized when the symmetry axis points to the center of mass of Earth. Any differential effect (including the violation signal, if any) would displace the centers of mass of the two cylinders relative to each other. An active control loop ensures that they remain centered: the control force itself contains the violation signal along with all classical (and GR) differential effects. In order to up-convert the frequency of the signal to higher frequency, the sensitive/symmetry axis must rotate relative to the satellite-to-Earth direction; hence, rotation must occur around an axis perpendicular to the symmetry axis of the cylinders. However, it is known in classical mechanics that a rotating axisymmetric rigid body is stable to small perturbations only if rotation occurs around the axis whose principal moment of inertia is distinct from the other two. The rotation mode of Microscope is slow and actively controlled, up to a maximum rate of about $\frac{1}{900} \mathrm{~Hz}$, with roughly a factor of 7 up-conversion from the orbital/signal frequency in the absence of rotation $[9,33]$.

In GG, the cylinders are allowed to move in the plane perpendicular to the symmetry axis (sensitive plane), where they respond to any differential acceleration: weak mechanical coupling in the plane, 2 degrees of freedom, and the effect of the violation signal maximized when the symmetry axis is perpendicular to the orbit plane. The violation signal would displace the test cylinders to a new equilibrium position, and the displacement is measured by a readout laser gauge. In accordance with the cylindrical symmetry of the system, rotation occurs around the symmetry axis, and it is stable; hence, passive attitude stabilization of the satellite is ensured (no active attitude control needed). At the same time, the signal is upconverted from the orbital frequency of $1.7 \times 10^{-4} \mathrm{~Hz}$ to the much higher rotation frequency of $1 \mathrm{~Hz}$ (with an upconversion factor of almost 6000) where electronic and thermal noise are much lower [31,34]. The rotation frequency is provided at the start of the mission and maintained by angular momentum conservation. Hence, the whole satellite spins with no need of motor and bearings, which are a well-known major source of noise for all rotating experiments in ground laboratories.

A rotating conductor in the magnetic field of Earth is known to slow down because of energy dissipation due to eddy currents induced in the conductor by a component of the magnetic field perpendicular to the spin axis. In GG, the largest such effect will take place in the Be test cylinder (the outer shell of the spacecraft will be manufactured in carbon fiber, and the inner Ti test cylinder has smaller size and lower conductivity). This effect has been calculated in [34] Sec. III $\mathrm{B}$, and found to be extremely small: the $Q$ factor of spin energy dissipation is $1.4 \times 10^{10}$, which means that in the total one-year duration of the mission, the spin frequency will decrease by about $1 \%$. This value has been obtained in the presence of a magnetic field of Earth reduced by a factor 150 by means of a $\mu$-metal shield and under worst-case assumptions. Any residual differential rotation between the outer shell of the GG spacecraft and its inner parts will be sensed and compensated, if needed, by means of the cold gas thrusters in charge of compensating for nongravitational forces (mostly drag from residual atmosphere).

Since the cylinders are suspended and coupled very weakly (taking advantage of the absence of weight in orbit), the frequencies of their normal modes are much lower than the spin frequency. This is a dynamical regime known as "supercritical rotation" (spin speed above the normal mode/ critical speed), which ensures "self-centering" better than achieved via construction and mounting by as much as the ratio of the spin-to-normal mode frequency squared. It is well known that such self-centering by physical laws requires 2 degrees of freedom; see Den Hartog [35], Chap. 6, particularly Eq. (6.2) for self-centering and Fig. 6.4 for evidence of rotation instability for systems with 1 degree of freedom. It cannot, therefore, be exploited in Microscope.

It is also well known that the presence of nonzero internal damping in the rotating system (rotating damping) gives rise to a slowly growing "whirling" motion at the normal mode frequency: the smaller the damping, the weaker the instability, the slower its growth, the smaller the fraction of the suspension force which is required to damp it (see [35] Sec. 7.4, [36] Sec. 4.5, and [37]). In GG, self-centering by physical laws is ensured at a few tens of $\mathrm{pm}$, and whirl is damped by capacitance sensors/actuators so as not to exceed a separation level between the centers of mass of $1.7 \mathrm{~nm}$, a noise well within the reach of capacitance bridges. Whirl damping is off during science data taking so that the test masses are totally passive save for the laser light of the interferometer in charge of reading their differential displacements.

Only differential accelerations between the test masses compete with the target violation signal. Accelerations caused by the primary body (Earth) and ascribed to GR have a specific dependence on the orbiting distance of each 
test mass; its first order differential effect is linear with the offset $\Delta r$ of the test masses in the direction to the center of mass of Earth, which may mimic a violation signal.

All effects predicted by GR on the test masses contain the very small dimensionless parameter,

$$
\epsilon=\frac{G M}{c^{2} r} \simeq 6.3 \times 10^{-10},
$$

where $G$ is the universal constant of gravity, $c$ the speed of light, $M$ the mass of Earth, and $r$ the orbital distance of the test body around it. The small parameter $\epsilon$ is the ratio between the Schwarzschild radius of Earth $G M / c^{2}=4.4 \mathrm{~mm}$ and the satellite orbital distance $r=R+h \simeq 7 \times 10^{6} \mathrm{~m}$.

The largest GR acceleration on each test mass $a_{E}$ was computed by Einstein [15] in 1915 for nonrotating interacting bodies [see, also, [28], Eq. (65a)]. The second largest one $a_{S_{1}}$ is due to the spin of the primary body [spin-orbit interaction; see [28], Eq. (65c)], while the smallest one $a_{S_{1} S_{2}}$ is due to the fact that both the primary body and the test mass are spinning [spin-spin interaction; see [28], Eq. (65d)]. Their respective orders of magnitude are

$$
\begin{gathered}
a_{E} \simeq 4 \epsilon g(h) \simeq 2 \times 10^{-8} \mathrm{~ms}^{-2}, \\
a_{S_{1}} \simeq 6 \epsilon \frac{S_{1}}{M} \frac{v}{r^{2}} \simeq 5.7 \times 10^{-10} \mathrm{~ms}^{-2}
\end{gathered}
$$

$\left(S_{1} \simeq 0.33 M R^{2} \omega_{1}\right.$ is the spin angular momentum of Earth with angular velocity $\omega_{1} \simeq 7.3 \times 10^{-5} \mathrm{rad} \mathrm{s}^{-1}$, hence, $\frac{S_{1}}{M} \simeq 9.8 \times 10^{8} \mathrm{~m}^{2} \mathrm{~s}^{-1} ; v \simeq 7.5 \times 10^{3} \mathrm{~m} \mathrm{~s}^{-1}$ is the orbital velocity of the satellite at distance $r \simeq 7 \times 10^{6} \mathrm{~m}$ ) and

$$
a_{S_{1} S_{2}} \simeq 3 \epsilon \frac{S_{1}}{M} \frac{S_{2}}{m} \frac{1}{r^{3}} \simeq 4.8 \times 10^{-22} \mathrm{~ms}^{-2}
$$

with $S_{2}$ the spin angular momentum of a test body of mass $m$. For the hollow test cylinders of GG spinning around the symmetry axis with angular velocity $\omega_{2}=2 \pi \mathrm{rad} \mathrm{s}^{-1}$, inner radius $a$, outer radius $b$, height $H$, it is $S_{2}=\frac{1}{2} m\left(a^{2}+b^{2}\right) \omega_{2}$; hence, in the worst case (largest value of $S_{2}$ ) of the outer cylinder ( $a \simeq 10.5 \mathrm{~cm}, b \simeq 13 \mathrm{~cm}$, $H \simeq 28.6 \mathrm{~cm}$ ), it is $\frac{S_{2}}{m} \simeq 0.088 \mathrm{~m}^{2} \mathrm{~s}^{-1}$.

What matters in UFF/WEP tests is the differential acceleration between the test masses. In the case of the GR effects (3) and (4), a differential acceleration arises because of a nonzero offset $\Delta r$ between the centers of mass of the test cylinders. In the case of GG, with $\Delta r \simeq 1.7 \mathrm{~nm}$ as reported above, we have

$$
\begin{aligned}
\Delta a_{E} & \simeq 3 a_{E} \frac{\Delta r}{r} \simeq 1.5 \times 10^{-23} \mathrm{~ms}^{-2}, \\
\Delta a_{S_{1}} & \simeq \frac{7}{2} a_{S_{1}} \frac{\Delta r}{r} \simeq 4.8 \times 10^{-25} \mathrm{~ms}^{-2} .
\end{aligned}
$$

Instead, the acceleration (5) depends on the geometrical properties of the test cylinders, which are necessarily different because they have been designed to be one inside the other, yielding a differential acceleration larger than the one caused by off-centering. A worst-case assumption is for the differential acceleration to be of the same order as the acceleration itself:

$$
\Delta a_{S_{1} S_{2}} \simeq a_{S_{1} S_{2}} \simeq 3 \times 10^{-22} \mathrm{~ms}^{-2} .
$$

Should the differential accelerations (6)-(8) not be identified as due to general relativity, they might be misinterpreted as a violation of UFF/WEP at the corresponding (spurious) levels:

$$
\begin{gathered}
\eta_{E}=\frac{\Delta a_{E}}{g(h)} \simeq 1.8 \times 10^{-24}, \\
\eta_{S_{1}}=\frac{\Delta a_{S_{1}}}{g(h)} \simeq 6 \times 10^{-26}, \\
\eta_{S_{1} S_{2}}=\frac{\Delta a_{S_{1} S_{2}}}{g(h)} \simeq 3.7 \times 10^{-23},
\end{gathered}
$$

showing that even the largest one caused by the spin-spin interaction is smaller that the GG target $\eta_{G G}=10^{-17}$ by more than 5 orders of magnitude. There is, therefore, no need to investigate the specific signature (e.g., the frequency, the phase, the dependence on the orbital parameters) and the exact values of these effects which come into play in the framework of general relativity.

In the case of Microscope, the spin-spin effect (11) is even less relevant than it is for GG because of a smaller value of $S_{2} / m$ (mostly because of a slower spin rate by almost 3 orders of magnitude) and also because of the lower precision target $\eta_{\text {microscope }}=10^{-15}$ of the mission. As for (9) and (10), they are about 60 times larger for Microscope (assuming $\Delta \mathrm{r} \simeq 0.1 \mu \mathrm{m}$ as reconstructed a posteriori); hence, their ratio to the mission target is about a factor of 2 smaller than the corresponding one for GG.

It is worth noticing that, as expected, the general relativistic effects considered above are much smaller than the Newtonian ones due to the nonzero quadrupole mass moments of Earth and the test masses.

The quadrupole mass moment of Earth $J_{2}^{(1)} \simeq 10^{-3}$ gives rise to an additional acceleration on each test mass [see, e.g., [28], Eq. (65e)]

$$
a_{Q_{1}} \simeq \frac{3}{2} g(h) J_{2}^{(1)}\left(\frac{R}{r}\right)^{2} \simeq 10^{-2} \mathrm{~ms}^{-2} .
$$

If the centers of mass of the test bodies are well centered on one another, its differential value is below the target. For GG we have 


$$
\Delta a_{Q_{1}} \simeq 4 a_{Q_{1}} \frac{\Delta r}{r} \simeq 10^{-17} \mathrm{~ms}^{-2},
$$

hence,

$$
\eta_{Q_{1}}=\frac{\Delta a_{Q_{1}}}{g(h)} \simeq 1.3 \times 10^{-18},
$$

which is a factor 8 smaller than the mission target $\eta_{G G}=10^{-17}$.

If each test cylinder has a nonzero quadrupole mass moment because its principal moments of inertia are not all equal, Earth's monopole does couple with it yielding an additional (Newtonian) acceleration which has been known as a major limitation to tests of UFF/WEP aiming for very high precision $[38,39]$. In the specific configuration of the GG experiment with principal moments of inertia $I_{z}$ relative to the spin/symmetry axis, $I_{x}=I_{y}$ relative to the Cartesian axes in the plane perpendicular to it, and a nonzero value of the ratio $\frac{\Delta I}{I_{x}}=\frac{I_{z}-I_{x}}{I_{x}}$, this effect has been calculated to be of the order (see [40], Sec. 2.2.5)

$$
a_{Q_{2}} \simeq \frac{3}{8} g(h) \frac{\Delta I}{I_{x}} \frac{r_{Q}^{2}}{r^{2}}
$$

with $r_{Q}^{2}=a^{2}+b^{2}+\frac{H^{2}}{3}$. The corresponding differential acceleration is dominated by the different value for the two test cylinders of the factor $\frac{\Delta I}{I_{x}} r_{Q}^{2}$. In GG, they have been designed so as to make this effect much smaller than the target signal,

$$
\Delta a_{Q_{2}} \simeq 5.6 \times 10^{-18} \mathrm{~ms}^{-2},
$$

yielding a (spurious) violation at the level

$$
\eta_{Q_{2}}=\frac{\Delta a_{Q_{2}}}{g(h)} \simeq 6.9 \times 10^{-19},
$$

which is about a factor 14 below the target. Note that this result has been obtained with a fractional difference in the moments of inertia for each test cylinder of the order of 0.01, which is not a demanding requirement at all.

Should GG aim at $10^{-18}$ with the same level of centering of the test masses, both effects (13) and (16) would be close to the target signal. However, their signature is different from that of the signal and known exactly from celestial mechanics [in the case of (13), the value of $J_{2}$ of Earth is well determined in satellite geodesy]. Hence, they can be separated from the signal by means of various measurements, each one to the target precision, in different dynamical conditions (e.g., different angles between the spin axis and the normal to the orbit plane). Many such measurements are possible because of the short integration time required by GG [34].
In the case of Microscope, a correct estimate of the effect (15) should be calculated taking into account the specific geometry and mass distribution of the test bodies. However, for the target $\eta_{\text {Microscope }}=10^{-15}$, this effect is not a matter of concern, and it has never been listed in the error budget of the mission.

\section{GENERAL RELATIVISTIC EFFECTS ON THE ORBITS OF THE TEST MASSES}

In a two-body problem, the secular effects due to general relativity on the semimajor axis of the orbit are zero. The orbital angular momentum vector (perpendicular to the orbit plane) and the Lenz vector (a vector pointing to the pericenter of the orbit whose modulus is the orbital eccentricity), which within Newtonian gravity are both fixed in inertial space, within GR are subjected to a secular precession with the same angular velocity. Since the precession velocity is the same, the orbit precesses as a whole [see [28], Eqs. (73) and (74)].

All GR contributions to orbit precession are proportional to the $\epsilon$ parameter (2). There is a contribution independent of rotation, a contribution due to the rotation of the primary body, and a contribution due to the rotation of both the primary and secondary body. We have

$$
\Omega_{E}^{\text {orbit }} \simeq 3 \epsilon n \simeq 2 \times 10^{-12} \operatorname{rad~s}^{-1}
$$

with $n$ the mean orbital angular velocity of the satellite [see [28], Eq. (76a)],

$$
\Omega_{S_{1}}^{\text {orbit }} \simeq 0.66 \epsilon\left(\frac{R}{r}\right)^{2} \omega_{1} \simeq 2.5 \times 10^{-14} \mathrm{rad} \mathrm{s}^{-1}
$$

[see [28], Eq. (76c) with $S_{1} \simeq 0.33 M R^{2} \omega_{1}$ ], and

$$
\begin{aligned}
\Omega_{S_{1} S_{2}}^{\text {orbit }} & \simeq \frac{3}{4} 0.33 \epsilon\left(\frac{R}{r}\right)^{2} \frac{a^{2}+b^{2}}{r^{2}} \frac{\omega_{1} \omega_{2}}{n} \\
& \simeq 3.16 \times 10^{-26} \mathrm{rad} \mathrm{s}^{-1}
\end{aligned}
$$

[see [28], Eq. (76d) with $S_{1} \simeq 0.33 M R^{2} \omega_{1}$ and $S_{2} \simeq$ $\frac{1}{2} m\left(a^{2}+b^{2}\right) \omega_{2}$ for the test cylinders of GG and a worstcase estimate].

Since UFF/WEP tests are differential experiments, only the differential precession of the orbits of the test cylinders relative to each other is relevant. For the orbit precessions (18) and (19), the difference between the two cylinders is due to the fact that they are not exactly at the same distance from the center of mass of Earth, hence,

$$
\Delta \Omega_{E}^{\text {orbit }} \simeq \frac{5}{2} \Omega_{E} \frac{\Delta r}{r} \simeq 1.2 \times 10^{-27} \mathrm{rad} \mathrm{s}^{-1}
$$

and 


$$
\begin{aligned}
\Delta \Omega_{S_{1}}^{\text {orbit }} & \simeq 3 \Omega_{S_{1}}^{\text {orbit }} \frac{\Delta r}{r} \simeq 1.8 \times 10^{-29} \mathrm{rad} \mathrm{s}^{-1}, \\
\Delta \Omega_{S_{1} S_{2}}^{\text {orbit }} & \simeq \frac{3}{4} 0.33 \epsilon\left(\frac{R}{r}\right)^{2} \frac{\omega_{1} \omega_{2}}{n} \frac{1}{r^{2}}\left(\Delta\left(a^{2}+b^{2}\right)\right) \\
& \simeq 2 \times 10^{-26} \mathrm{rad} \mathrm{s}^{-1} .
\end{aligned}
$$

In addition, it has been shown [[28], Eq. (46)] that according to GR, the quadrupole mass moment of the primary body gives an additional contribution to the precession of the orbit of the test mass:

$$
\Omega_{Q_{1} G R}^{\text {orbit }} \simeq \frac{9}{4} \epsilon J_{2}^{(1)}\left(\frac{R}{r}\right)^{2} n \simeq 1.4 \times 10^{-15} \mathrm{rad} \mathrm{s}^{-1} .
$$

The corresponding differential precession between the orbits of the test masses, with the center of mass offset as in the case of GG, is

$$
\Delta \Omega_{Q_{1} G R}^{\text {orbit }} \simeq \frac{7}{2} \Omega_{Q_{1} G R} \frac{\Delta r}{r} \simeq 1.17 \times 10^{-30} \mathrm{rad} \mathrm{s}^{-1} .
$$

These results show that the largest differential precession predicted by general relativity is $\Delta \Omega_{S_{1} S_{2}}^{\text {orbit }}$ due to the proper rotation of Earth coupling with the proper rotation of the test cylinders. However, the angular velocity of differential precession is extremely small, with a period more than 8 orders of magnitude longer than the age of the Universe.

By comparison with the GR effects computed above, it is worth recalling the Newtonian contributions to orbit precession which are already taken into account in the experiments and their numerical simulations. The largest one is the well-known precession due to the quadrupole mass moment of Earth on any point mass moving on an inclined orbit around it. This effect is exploited by both Microscope and GG in order to keep the satellite in a sunsynchronous orbit (with an appropriate choice of the inclination for the selected altitude). It is given by

$$
\Omega_{Q_{1}}^{\text {orbit }} \simeq \frac{3}{2} J_{2}^{(1)}\left(\frac{R}{r}\right)^{2} n \simeq 1.4 \times 10^{-6} \mathrm{rad} \mathrm{s}^{-1},
$$

yielding a much smaller differential precession between the orbits of the test masses:

$$
\Delta \Omega_{Q_{1}}^{\text {orbit }} \simeq \frac{7}{2} \Omega_{Q_{1}} \frac{\Delta r}{r} \simeq 1.2 \times 10^{-21} \mathrm{rad} \mathrm{s}^{-1} .
$$

It has been shown in [29], Eq. (71) that an additional Newtonian precession of the orbit occurs if the secondary body has a nonzero quadrupole mass moment of its own, coupling with the monopole of Earth. In this case, the dependence on the average size of the test body (squared) makes the effect many orders of magnitude smaller than the previous one. With the typical numbers of GG, we have for the largest body,

$$
\Omega_{Q_{2}}^{\text {orbit }} \simeq \frac{3}{2} J_{2}^{(2)}\left(\frac{\bar{r}}{r}\right)^{2} n \simeq 6.4 \times 10^{-21} \mathrm{rad} \mathrm{s}^{-1},
$$

where $J_{2}^{(2)}$ is the quadrupole mass moment of the test body (defined similarly to the quadrupole mass moment $J_{2}^{(1)}$ of Earth in the expansion of its gravitational field in multipole mass moments), and $\bar{r}$ is the average size (half the sum of the inner and outer radius of the test cylinder). The numerical estimate refers to GG (worst-case value), and the corresponding differential precession turns out to be only 1 order of magnitude smaller:

$$
\Delta \Omega_{Q_{2}}^{\text {orbit }} \simeq 7.5 \times 10^{-22} \mathrm{rad} \mathrm{s}^{-1},
$$

a value slightly smaller than the differential precession (27) due to the quadrupole mass moment of Earth.

As we can see by comparing (18) and (26), the largest precession due to GR is 6 orders of magnitude smaller than the largest Newtonian precession, while in the case of differential precession, the Newtonian ones dominate by 4 orders of magnitude [see (27) and (29) in comparison with the largest GR differential precession (23)].

In order to assess how much these orbit precessions affect the test, we use the variation of the elements' perturbative equations in the form of Gauss (see [41]), in which the time variation of the orbital elements are expressed in terms of the radial, transverse, and out-ofplane components of the perturbing acceleration $a_{R}, a_{T}$, and $a_{W}$, respectively. For the effects on the pericenter and the node, we use Eqs. (3.43) and (3.47) of [41] and find the following relationships between a (differential) orbit precession rate and the corresponding (differential) components of the perturbing acceleration that generates it:

$$
\begin{gathered}
\Delta a_{W} \simeq v \Delta \Omega_{Q_{2}}^{\text {orbit }} \simeq 5.6 \times 10^{-18} \mathrm{~ms}^{-2}, \\
\Delta a_{R} \simeq \Delta a_{T} \simeq e v \Delta \Omega_{Q_{2}}^{\text {orbit }} \simeq 5.6 \times 10^{-20} \mathrm{~ms}^{-2} .
\end{gathered}
$$

As expected, they are related through the orbital velocity $v$, and for the same precession of the orbit, the radial and transverse components of the perturbation involved are smaller than the out-of-plane component by as much as the orbital eccentricity $e$ (we have used the maximum value required for $\mathrm{GG} e \lesssim 0.01$; for Microscope, the requirement is $e \lesssim 0.005$ ).

Only the radial component $\Delta a_{R}$ would compete with the violation signal. The corresponding spurious contribution to violation comes from the Newtonian differential precession and amounts to

$\eta_{\text {orbit-precession }}=\frac{\Delta a_{R}}{g(h)} \simeq \frac{5.6 \times 10^{-20}}{8.1} \simeq 6.9 \times 10^{-21}$, 
which is more than 3 orders of magnitude smaller than the GG target. As for the contribution from the largest GR effect (22), it is 4 orders of magnitude smaller still.

\section{GENERAL RELATIVISTIC EFFECTS ON THE SPIN AXES OF THE TEST MASSES}

In the framework of general relativity, the spin axes of the test masses are subjected to the following precessions:

$$
\Omega_{E}^{\text {spinaxis }} \simeq \frac{3}{2} \epsilon n \simeq 10^{-12} \mathrm{rad} \mathrm{s}^{-1}
$$

caused by the primary body (Earth) regardless of its rotation [also known as de Sitter precession; see, e.g., [28], Eq. (42)] and

$$
\Omega_{S_{1}}^{\text {spinaxis }} \simeq \frac{0.33}{2} \epsilon\left(\frac{R}{r}\right)^{2} \omega_{1} \simeq 6.3 \times 10^{-15} \mathrm{rad} \mathrm{s}^{-1}
$$

due to the proper rotation of Earth with spin angular momentum $S_{1} \simeq 0.33 M R^{2} \omega_{1}$ [also known as LenseThirring precession; see, e.g., [28], Eq. (29)]. The corresponding differential precessions between the spin axes of the two cylinders, due to the fact that they are not exactly centered on each other, are (GG case)

$$
\Delta \Omega_{E}^{\text {spinaxis }} \simeq \frac{5}{2} \Omega_{E}^{\text {spinaxis }} \frac{\Delta r}{r} \simeq 6.2 \times 10^{-28} \mathrm{rad} \mathrm{s}^{-1}
$$

and

$$
\Delta \Omega_{S_{1}}^{\text {spinaxis }} \simeq 3 \Omega_{S_{1}}^{\text {spinaxis }} \frac{\Delta r}{r} \simeq 4.6 \times 10^{-30} \mathrm{rad} \mathrm{s}^{-1} .
$$

By far, a larger precession of the spin axes of the test cylinders is Newtonian, due to the fact that they have a nonzero fractional difference of their principal moments of inertia $\frac{I_{z}-I_{x}}{I_{x}}=\frac{\Delta I}{I_{x}}, z$ being the direction of the symmetry/ rotation axis. According to [29], Eq. (47), the precession rate of the spin axis is

$$
\Omega_{Q_{2} S_{2}}^{\text {spinaxis }} \simeq \frac{1}{2} \frac{\Delta I}{I_{x}} \frac{n^{2}}{\omega_{2}} \simeq 1.3 \times 10^{-9} \mathrm{rad} \mathrm{s}^{-1},
$$

where the numerical estimate refers to the GG test cylinders (worst case: $\frac{\Delta I}{I_{x}} \simeq 0.014$ ). Note that this angular precession rate is proportional to the ratio $\frac{n^{2}}{\omega_{2}}$ between the orbital mean motion $n$ squared and the rotation angular velocity of the test cylinder $\omega_{2}$, thus, implying that for test cylinders with the same orbital velocity, those which spin faster have a slower precession rate (the ratio of the spin rates is about 900 to 1 between GG and Microscope). The corresponding differential precession between the test cylinders is not much smaller because they cannot have values of $\frac{\Delta I}{I_{x}}$ exactly (or very nearly) equal. In GG, by requiring that the relative difference is $2 \times 10^{-3}$, the differential precession rate of the spin axes is

$$
\Delta \Omega_{Q_{2} S_{2}}^{\text {spinaxis }} \simeq \frac{1}{2} \Delta\left(\frac{\Delta I}{I_{x}}\right) \frac{n^{2}}{\omega_{2}} \simeq 1.8 \times 10^{-10} \mathrm{rad} \mathrm{s}^{-1}
$$

with a differential precession period of $1000 \mathrm{yr}$. In the planned one-year duration of the mission, the differential precession angle amounts to about $0.3^{\circ}$, while each spin axis precesses by about $2.4^{\circ}$. We recall that in GG, the test cylinders spin around their symmetry axes; hence, the rotation is stable against small perturbations. In addition, the spin frequency is higher than the normal mode frequencies in the plane perpendicular to the spin/symmetry axis: a dynamical condition which is known to ensure natural damping of the conical modes (precessions) (see $[36,42])$.

A realistic estimate of spin axes precessions in the case of Microscope would require knowledge of the geometrical and mass properties of the test cylinders and of the rotation control of the system. However, it is apparent that the Newtonian framework under which the experiment has been designed is fully adequate to the task, since precessions due to general relativity are many orders of magnitude smaller than the Newtonian ones.

\section{EFFECTS ON THE READOUT LASER GAUGE}

In GG, the relative displacements of the test cylinders in the sensitive plane perpendicular to the spin/symmetry axis are read by a laser interferometry gauge. It was proposed in 2010 by Shao [43] (Jet Propulsion Laboratory) as a substitution of the originally planned capacitance readout (tested in the laboratory prototype of GG) because of its numerous advantages. A violation signal at the target level of $10^{-17}$ shows up as a $0.6 \mathrm{pm}$ displacement between the centers of mass of the test cylinders pointing to (or away from) the center of mass of Earth at the spin frequency of $1 \mathrm{~Hz}$ (after up-conversion by rotation from the much lower orbital frequency of $1.7 \times 10^{-4} \mathrm{~Hz}$ ). The laser gauge is expected to have a displacement noise of $\frac{1 \mathrm{pm}}{\sqrt{\mathrm{Hz}}}$ at $1 \mathrm{~Hz}$.

In a spinning experiment, the readout is obviously corotating with the system. As reported in Sec. I, concerns have been expressed by the SARP panel of ESA about the effects of rotation (of the tests cylinders and Earth) on the readout laser gauge.

The laser gauge designed for GG is presented in [44] where some key sources of noise are discussed on the basis of the results of specific lab tests. In [45], a measured displacement noise of $\frac{3 \mathrm{pm}}{\sqrt{\mathrm{Hz}}}$ is reported at $1 \mathrm{~Hz}$, and the onset of a spurious displacement in the presence of rotation (Sagnac effect) is discussed and quantified. The issue is as follows: if-from the point of separation to the point of recombination-the interfering laser rays happen to 
enclose a nonzero area, and the axis normal to this area has a component along the rotation axis, then the laser rays traveling in the sense of rotation and those traveling opposite to it (both at the speed of light) do have different flight times, yielding a spurious interference signal. In laser gyros, this "spurious" signal is used to measure the rotation angular velocity perpendicular to the area enclosed by the gyro (e.g., the diurnal rotation velocity of Earth).

In the case of GG, the laser rays are (nominally) aligned, and the area enclosed from separation to recombination is zero; hence, there should be no spurious displacement. However, a nonzero area arises in the presence of a misalignment. As shown in [45], a misalignment of $10 \mu \mathrm{m}$ (with a typical $20 \mathrm{~cm}$ separation from beam launcher to target) results in a spurious displacement of $\simeq 4 \times 10^{-14} \mathrm{~m}$. It is 2 orders of magnitude smaller than the signal; moreover, only its time variation at the spin frequency competes with the violation signal (a constant bias does not matter). There is even a better way out: the lasers can be arranged in such a way that the angular velocity vector of rotation has no component perpendicular to the nonzero area resulting from the misalignment, and, therefore, there is (nominally) no Sagnac effect. In reality, the effect is reduced even further. This strategy has been followed from the start in the design of the GG laser gauge.

As far as the effects of the rotation of Earth on the laser gauge of GG are concerned, it is known that the rotation of a celestial body (and also its flattening) do affect the angle of deviation and the propagation time of light rays, which in their journey from emitter to receiver, happen to pass close to the body (see, e.g., [46]). In high-precision astronomical measurements such as those carried out by the GAIA mission of ESA, these effects are indeed carefully calculated and taken into account in the framework of general relativity. However, in GG there is no measurement over astronomical distances: the laser interferometer works inside the spacecraft, with optical path differences (in between the targets located, respectively, on the outer surface of the inner cylinder and on the inner surface of the outer one) of $2 \mathrm{~cm}$ or less and a path length of about $20 \mathrm{~cm}$

We, therefore, do not expect that any effect from the spin (and flattening) of Earth on the laser interferometry readout of GG should be taken into account. More important, there is recent experimental evidence which supports this conclusion. A heterodyne laser gauge similar to the one of GG, though more demanding and complex, has recently flown on the LISA Pathfinder (LPF) [47]. In this case, the optical path difference is $\simeq 38 \mathrm{~cm}$, which is the separation distance between the test masses that constitute the mirrors, which, in the final gravitational wave interferometer LISA, will be located in different spacecraft about $5 \times 10^{6} \mathrm{~km}$ away from each other. The laser gauge of LPF has been designed to achieve low noise down to $7 \pm 10^{-4} \mathrm{~Hz}$ (while in GG the violation signal is up-converted by rotation to $1 \mathrm{~Hz}$ ). Reaching this level of noise at such low frequencies and over $38 \mathrm{~cm}$ separation of the test masses requires, among other things, the frequency of the laser to be stabilized. This is a demanding requirement in space, which does not apply in the case of GG because of the $1 \mathrm{~Hz}$ frequency of the signal and $2 \mathrm{~cm}$ maximum separation. The displacement noise as measured by LPF above $0.06 \mathrm{~Hz}$ is reported to be $\frac{0.035 \mathrm{pm}}{\sqrt{\mathrm{Hz}}}$ (about 30 times lower than required for $\mathrm{GG}$ at $1 \mathrm{~Hz}$ ); it is interpreted by the authors as entirely due to the interferometer, and no evidence is reported of any effect due to the rotation and flattening of Earth [47]. In addition, it turns out to be about 100 times lower in the absence of weight than obtained in ground tests before launch.

\section{CONCLUSIONS}

The Microscope satellite launched on April 25, 2016 is testing the universality of free fall and the weak equivalence principle in the gravitational field of Earth aiming at 2 orders of magnitude improvement over the current best tests. In a similar orbit but with a different experiment design, the GG satellite aims for a test 100 times better than Microscope. They must detect extremely small differential accelerations acting between test cylinders of different composition, ruling out any competing effect which is due to known physics.

In 2015, during the evaluation process of the space mission proposals short listed as candidates for the medium size mission M4 of the European Space Agency, the panel appointed by the agency to evaluate GG made the point that while the experiment has been designed in the framework of Newtonian physics, it should instead take into account general relativistic effects, in particular, those which involve the spin angular momentum of the source body (Earth) and that of the test cylinders.

We have carefully analyzed all known general relativistic effects on the test cylinders of GG and Microscope showing that they are all negligible by and large.

\section{ACKNOWLEDGMENTS}

Thanks are due to Norbert Wex, David Lucchesi, Angelo Tartaglia, Slava Turyshev, Juergen Mueller, Alberto Anselmi, and Maria Teresa Crosta for their contributions. Support from the European Space Agency in the development of a low noise laser gauge over small distances is gratefully acknowledged. 
[1] A. M. Nobili, D. M. Lucchesi, M. T. Crosta, M. Shao, S. G. Turyshev, R. Peron, G. Catastini, A. Anselmi, and G. Zavattini, On the universality of free fall, the equivalence principle, and the gravitational redshift, Am. J. Phys. 81, 527 (2013).

[2] A. Einstein, Grundlage der allgemeinen Relativitätstheorie, Ann. Phys. (Berlin) 49, 769 (1916); The foundation of the general theory of relativity, in The Principle of Relativity (Dover, New York, 1952).

[3] S. Baeßler, B. R. Heckel, E. G. Adelberger, J. H. Gundlach, U. Schmidt, and H.E. Swanson, Improved Test of the Equivalence Principle for Gravitational Self-Energy, Phys. Rev. Lett. 83, 3585 (1999).

[4] S. Schlamminger, K.-Y. Choi, T. A. Wagner, J. H. Gundlach, and E. G. Adelberger, Test of the Equivalence Principle Using a Rotating Torsion Balance, Phys. Rev. Lett. 100, 041101 (2008).

[5] T. A. Wagner, S. Schlamminger, J. Gundlach, and E. G. Adelberger, Torsion-balance tests of the weak equivalence principle, Classical Quantum Gravity 29, 184002 (2012).

[6] J. G. Williams, S. G. Turyshev, and D. H. Boggs, Progress in Lunar Laser Ranging Tests of Relativistic Gravity, Phys. Rev. Lett. 93, 261101 (2004).

[7] J. G. Williams, S. G. Turyshev, and D. H. Boggs, Lunar laser ranging tests of the equivalence principle, Classical Quantum Gravity 29, 184004 (2012).

[8] J. Mueller, F. Hoffmann, and L. Biskupek, Testing various facets of the equivalence principle using lunar laser ranging, Classical Quantum Gravity 29, 184006 (2012).

[9] P. Touboul, G. Metris, V. Lebat, and A. Robert, The MICROSCOPE experiment, ready for the in-orbit test of the equivalence principle, Classical Quantum Gravity 29, 184010 (2012).

[10] A. M. Nobili, M. Shao, R. Pegna, G. Zavattini, S. G. Turyshev, D. M. Lucchesi, A. De Michele, S. Doravari, G. L. Comandi, T. R. Saravanan, F. Palmonari, G. Catatstini, and A. Anselmi, 'Galileo Galilei' (GG): Space test of the weak equivalence principle to $10^{-17}$ and laboratory demonstrations, Classical Quantum Gravity 29, 184011 (2012).

[11] L. Thou, S. Long, B. Tang, X. Chen, F. Gao, W. Peng, W. Duan, J. Zhong, Z. Xiong, J. Wang, Y. Zhang, and M. Zhan, Test of Equivalence Principle at $10^{-8}$ Level by a DualSpecies Double-Diffraction Raman Atom Interferometer, Phys. Rev. Lett. 115, 013004 (2015).

[12] A. M. Nobili, Fundamental limitations to high-precision tests of the universality of free fall by dropping atoms, Phys. Rev. A 93, 023617 (2016).

[13] G. Arnold, A. Bazzano, P. Binetruy, E. Chassefiere, M. Diaz Trigo, J. Drew, E. Friis-Christensen, O. Grasset, G. Harendel, C. Haswell, M. Hewitson, G. Nelemans, B. Nordstrom, W. Percival, G. Peres, J. Rodriguez-Pacheco Martin, M. Sauvage, B. Stelzer, E. Szuszkiewicz, R. Tucker, and J. Zarnecki, Report to the European Space Agency on the GG proposal for the medium size mission M4 (2015).

[14] A. A. Michelson and E. W. Morley, On the relative motion of the Earth and the luminiferous ether, Am. J. Sci. 34, 333 (1887).

[15] A. Einstein, Erklarung der Perihelwegung Merkur der allgemeinen Relativitatstheorie, in Akademie-Vorträge: Sitzungsberichte der Preußischen Akademie der Wissenschaf- ten (Wiley-VCH Verlag GmbH \& Co, Weinheim, 1915), pp. 831-839; Explanation of the Perihelion Motion of Mercury from the General Theory of Relativity, translated by A. Engel, The Collected Papers of Albert Einstein Vol. 6 (Princeton University Press, Princeton, 1996), p. 112 .

[16] A. Einstein, Die Feldgleichungen der Gravitation, Preussische Akademie der Wissenschaften, Sitzungsberichte part 2 Wiley-VCH Verlag $\mathrm{GmbH} \& \mathrm{Co}$, Weinheim, 1915), pp. 844-847; The Field Equations of Gravitation, translated by A. Engel, The Collected Papers of Albert Einstein Vol. 6 (Princeton University Press, Princeton, 1996), p. 117.

[17] W. de Sitter, Planetary motion and the motion of the moon according to Einstein's theory, in Proceedings of the Royal Netherlands Academy of Arts and Sciences, Amsterdam (Royal Netherlands Academy of Arts and Sciences, Amsterdam, 1917), Vol. 19, p. 317, http://www.dwc.knaw.nl/ DL/publications/PU00012364.pdf.

[18] H. Thirring, Über die Wirkung rotierender ferner Massen in der Einsteinschen Gravitationstheorie (On the effect of rotating distant masses in Einstein's theory of gravitation), Phys. Z. 19, 33 (1918).

[19] J. Lense and H. Thirring, Über den Einfluss der Eigenrotation der Zentralkörper auf die Bewegung der Planeten und Monde nach der Einsteinschen Gravitationstheorie (On the influence of the proper rotation of central bodies on the motions of planets and moons according to Einstein's theory of gravitation), Phys. Z. 19, 156 (1918).

[20] B. Mashhoon, F. W. Hehl, and D. S. Theiss, On the gravitational effects of rotating masses: The Thirring-Lense papers, Gen. Relativ. Gravit. 16, 711 (1984).

[21] M. Mathisson, Neue Mechanik materieller Systeme, Acta Phys. Pol. 6, 163 (1937); New mechanics of material systems, Gen. Relativ. Gravit. 42, 1011 (2010).

[22] A. Papapetrou, Spinning test-particles in general relativity I, Proc. R. Soc. A 209, 248 (1951).

[23] W. G. Dixon, A covariant multipole formalism for extended test bodies in general relativity, Nuovo Cimento 34, 317 (1964).

[24] L. Schiff, Possible New Experimental Tests of General Relativity Theory, Phys. Rev. Lett. 4, 215 (1960).

[25] L. Schiff, Motion of a gyroscope according to Einstein's theory of gravitation, Proc. Natl. Acad. Sci. U.S.A. 46, 871 (1960).

[26] L. Schiff, Proceedings of the Conference on Experimental Tests of Theories of Relativity, Stanford, 1961.

[27] C. W. F. Everitt et al., Gravity Probe B: Final Results of a Space Experiment to Test General Relativity, Phys. Rev. Lett. 106, 221101 (2011).

[28] B. M. Barker and R.F. O'Connell, Derivation of the equations of motion of a gyroscope from the quantum theory of gravitation, Phys. Rev. D 2, 1428 (1970).

[29] B. M. Barker and R. F. O'Connell, Gravitational two-body problem with arbitrary masses, spins and quadrupole moments, Phys. Rev. D 12, 329 (1975).

[30] E. G. Adelberger, J. H. Gundlach, B. R. Heckel, S. Hoedl, and S. Schlamminger, Torsion balance experiments: A low-energy frontier of particle physics, Prog. Part. Nucl. Phys. 62, 102 (2009). 
[31] R. Pegna, A. M. Nobili, M. Shao, S. G. Turyshev, G. Catastini, A. Anselmi, R. Spero, S. Doravari, G. L. Comandi, and A. De Michele, Abatement of Thermal Noise due to Internal Damping in 2D Oscillators with Rapidly Rotating Test Masses, Phys. Rev. Lett. 107, 200801 (2011).

[32] CNES-ONERA press release, September 27, 2016, https:// presse.cnes.fr/en/cnes-onera-cooperation-first-ultra-precisemeasurements-microscope.

[33] P. Touboul, in Proceedings of Microscope Colloqium IV, Paris, 2015, http://microscope4.sciencesconf.org/81163.

[34] A. M. Nobili, R. Pegna, M. Shao, S. G. Turyshev, G. Catastini, A. Anselmi, R. Spero, S. Doravari, G. L. Comandi, D. M. Lucchesi, and A. De Michele, Integration time in space experiments to test the equivalence principle, Phys. Rev. D 89, 042005 (2014).

[35] J. P. Den Hartog, Mechanical Vibrations (Dover Publications Inc., New York, 1985).

[36] G. Genta, Vibrations of Structures and Machines (SpringerVerlag, New York, 1993).

[37] A. M. Nobili, D. Bramanti, E. Polacco, G. Catastini, A. Anselmi, S. Portigliotti, A. Lenti, P. di Giamberardino, S. Monaco, and R. Ronchini, Evaluation of a proposed test of the weak equivalence principle using Earth-orbiting bodies in high-speed co-rotation: Re-establishing the physical bases, Classical Quantum Gravity 16, 1463 (1999).
[38] J. P. Blaser et al., Report No. ESA/NASA SCI (93)4, 1993.

[39] J. P. Blaser et al., Report No. ESA SCI (96)5, 1996.

[40] A. M. Nobili et al., GG Phase A Study (Agenzia Spaziale Italiana, Rome, 2000).

[41] A. Milani, A. M. Nobili, and P. Farinella, Non-Gravitational Perturbations and Satellite Geodesy (Adam Hilger Ltd., Bristol, 1987).

[42] A. M. Nobili, D. Bramanti, G. Catastini, E. Polacco, G. Genta, E. Brusa, V. P. Mitrofanov, A. Bernard, P. Touboul, A. J. Cook, J. Hough, I. W. Roxburgh, A. Polnarev, W. Flury, F. Barlier, and C. Marchal, Proposed noncryogenic, nondrag-free test of the equivalence principle in space, New Astron. 3, 175 (1998).

[43] M. Shao, SIM-like laser gauge and adaptation to GG, $G G$ Workshop, Pisa, 2010.

[44] M. Pisani, G. Mana, and A. M. Nobili, Design of an interferometric displacement sensor with picometer resolution for the Galileo Galilei mission, in Proceedings of Metrology for Aerospace, Benevento, 2015, p. 591.

[45] M. Pisani, M. Zucco, and A. M. Nobili, Laboratory tests of a high-precision laser interferometry readout for the GG experiment in space, in Proceedings of Metrology for Aerospace, Firenze, 2016, p. 260.

[46] S. A. Klioner, Influence of the quadrupole field and rotation of objects on light propagation, Sov. Astron. 35, 523 (1991).

[47] M. Armano et al., Sub-Femto- $g$ Free Fall for Space-Based Gravitational Wave Observatories: LISA Pathfinder Results, Phys. Rev. Lett. 116, 231101 (2016). 